\title{
Toxoplasma gondii infection in interstate truck drivers: a case-control seroprevalence study
}

Cosme Alvarado-Esquivel ${ }^{1 *}$, Sandy Janet Pacheco-Vega' ${ }^{1}$, Jesús Hernández-Tinoco², Misael Salcedo-Jáquez', Luis Francisco Sánchez-Anguiano², Luis Omar Berumen-Segovia', Elizabeth Rábago-Sánchez ${ }^{1,3}$ and Oliver Liesenfeld ${ }^{4,5}$

\begin{abstract}
Background: Infection with Toxoplasma gondii can be acquired via the ingestion of undercooked or raw meat containing tissue cysts, or via ingestion of water contaminated with oocysts. Professional long distance truck driving may have epidemiological importance for food-borne infections since drivers eat out of home and in places where hygiene and cooking practices are uncertain. We aimed to determine whether interstate truck drivers in Durango, Mexico have an increased risk of infection with T. gondii as indicated by seropositivity; and to determine the socio-demographic, work, clinical, and behavioral characteristics associated with T. gondii seropositivity in interstate truck drivers.
\end{abstract}

Methods: Through a case-control study design, 192 truck drivers and 192 controls from the general population of the same region matched by gender and age were examined with enzyme-linked immunoassays for the presence of anti-Toxoplasma lgG and IgM antibodies. Socio-demographic, work, clinical and behavioral characteristics from the truck drivers were obtained.

Results: Anti-T. gondii lgG antibodies were found in 23 (12.0\%) of 192 truck drivers and in 13 (6.8\%) of 192 controls $(\mathrm{OR}=21.0 ; 95 \% \mathrm{Cl}: 1.23-358.38 ; P=0.002)$. Anti-T. gondii IgM antibodies were found in 7 (3.6\%) cases and in $7(3.6 \%)$ controls $(P=1.00)$. The seroprevalence of $T$. gondii infection was higher in drivers with reflex impairment than in those without this impairment $(4 / 13,30.8 \%$ vs $19 / 179,10.6 \%$, respectively; $P=0.05)$, and in drivers with hearing impairment than in those without this impairment $(3 / 7,42.9 \%$ vs $20 / 185,10.8 \%$, respectively; $P=0.03)$. Multivariate analysis of work and behavioral characteristics of truck drives showed positive associations of $T$. gondii exposure with trips to the south of Mexico $(\mathrm{OR}=3.11 ; 95 \% \mathrm{Cl}$ : 1.02-9.44; $P=0.04)$ and consumption of horse meat $(\mathrm{OR}=5.18$; 95\% Cl: 1.62-16.55; $P=0.005)$.

Conclusions: Results suggest that interstate truck drivers may have an increased risk for $T$. gondii infection, and that T. gondii exposure may be impacting neurological functions in truck drivers. Contributing factors for T. gondii exposure should be taken into account for the design of optimal prevention measures against $T$. gondii infection.

Keywords: Toxoplasma gondii, Seroprevalence, Truck drivers, Case-control study, Risk factors

\footnotetext{
* Correspondence: alvaradocosme@yahoo.com

${ }^{1}$ Faculty of Medicine and Nutrition, Juárez University of Durango State,

Avenida Universidad S/N, 34000 Durango, Dgo, Mexico

Full list of author information is available at the end of the article
}

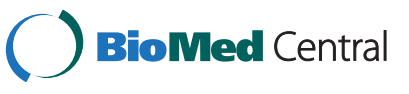

C 2015 Alvarado-Esquivel et al.; licensee BioMed Central. This is an Open Access article distributed under the terms of the Creative Commons Attribution License (http://creativecommons.org/licenses/by/4.0), which permits unrestricted use, distribution, and reproduction in any medium, provided the original work is properly credited. The Creative Commons Public Domain Dedication waiver (http://creativecommons.org/publicdomain/zero/1.0/) applies to the data made available in this article, unless otherwise stated. 


\section{Background}

The protozoan parasite Toxoplasma gondii (T. gondii) causes infections in humans all around the world [1]. Most infections with $T$. gondii are acquired by ingesting food or water contaminated with $T$. gondii oocysts shed by cats or by eating undercooked or raw meat containing $T$. gondii tissue cysts [2]. Infections with $T$. gondii are usually asymptomatic but in some individuals infections may lead to severe disease. Toxoplasmosis is characterized by affection of the host's lymph nodes, eyes, central nervous system, and other organs [2-5]. Infections with $T$. gondii have been associated with schizophrenia [6,7], traffic accidents [8], work accidents [9], and suicide attempts $[10,11]$.

Interstate truck drivers are a group of workers driving trucks for long distances from one state to the other within a country and some of them drive trucks abroad too. This condition may have epidemiological importance for food-borne infectious diseases because such workers eat out of home in places where hygiene and cooking practices are uncertain. Eating away of home has been associated with $T$. gondii exposure in Mexico [12]. In a previous study in patients with liver disease, we observed that patients with an occupation of truck driver had a higher seroprevalence of $T$. gondii infection than those without this occupation [13]. However, casecontrol studies to assess the association of $T$. gondii exposure and interstate truck driver occupation have not been performed. Therefore, we performed an age- and gender matched case-control study to determine the association of $T$. gondii seropositivity with interstate truck driver occupation in Durango, Mexico. Furthermore, we investigated the socio-demographic, work, clinical, and behavioral characteristics associated with $T$. gondii seropositivity in the interstate truck drivers studied.

\section{Methods \\ Study design and study populations}

Through a case-control study design, we studied the association of seropositivity to $T$. gondii with interstate truck drivers. The present study was performed in Durango City, Mexico from November 2013 to July 2014.

Interstate truck drivers: One hundred and ninety two interstate truck drivers were enrolled in the study. Inclusion criteria for the cases were current working as interstate truck drivers for at least one year, aged 18 years and older, any gender, and who accepted to participate in the study. As a strategy to enroll drivers, they were invited to participate in gas stations, small motels for truck drivers, and truck stops on the roads. One hundred and eighty seven of them were males and five were female. The mean age of the drivers was $43.40 \pm 10.16$ years (range: 20-71 years).
Control subjects: One hundred and ninety two control subjects without truck driver occupation matched with drivers by age and gender were included in the study. Control subjects were randomly selected from the general population of Durango City, Mexico. The mean age in controls was $43.45 \pm 10.21$ (range: $20-71$ years) and comparable with that in truck drivers $(P=0.96)$.

\section{Socio-demographic, clinical, work and behavioral data}

We obtained the socio-demographic, work, clinical and behavioral characteristics of the participants with the aid of a standardized questionnaire. Socio-demographic items included age, gender, birthplace, residence area, educational level, and socio-economic level. Work characteristics assessed in truck drivers included number of years in the activity, type of materials transported, frequency and duration of trips, regions and number of states visited within Mexico, work abroad, traffic accidents, type of places of getting meals out of home, transportation of animals, and washing the truck by themselves. Clinical data in truck drivers included presence of any disease, presence or history of lymphadenopathy, frequent headache, dizziness, impairments in memory, reflexes, hearing and vision, and history of blood transfusion, transplant or surgery. Behavioral items included contact with animals or cat feces, type of meat consumed, consumption of raw or undercooked meat, unpasteurized milk, dried or cured meat, consumption of unwashed raw vegetables, fruits, or untreated water, frequency of eating away from home (in restaurants or fast food outlets), washing hands before eating, contact with soil, and type of flooring at home.

\section{Laboratory tests}

A blood sample from each participant was obtained. Blood samples were centrifuged and sera were obtained and kept frozen at $-20^{\circ} \mathrm{C}$ until analyzed. Sera were analyzed by qualitative and quantitative methods for anti- $T$. gondii IgG antibodies with the commercially available enzyme immunoassay kit "Toxoplasma IgG" (Diagnostic Automation Inc., Calabasas, CA, USA). Anti-T. gondii IgG antibody levels were expressed as International Units $(\mathrm{IU}) / \mathrm{ml}$, and a result equal to or greater than $8 \mathrm{IU} / \mathrm{ml}$ was considered positive. In addition, sera positive for anti- $T$. gondii IgG antibodies were further analyzed for anti-T. gondii IgM antibodies by the commercially available enzyme immunoassay "Toxoplasma IgM" kit (Diagnostic Automation Inc., Calabasas, CA, USA). All tests were performed following the instructions of the manufacturer.

\section{Statistical analysis}

We performed the statistical analysis with the aid of Epi Info version 7 and SPSS 15.0 (SPSS Inc. Chicago, 
Illinois). For calculation of the sample size, we used a $95 \%$ confidence level, a power of $80 \%$, a $1: 1$ proportion of cases and controls, a reference seroprevalence of $6.1 \%$ [14] as the expected frequency of exposure in controls, and an odds ratio of 2.8. The result of the sample size calculation was 175 cases and 175 controls. Age among the groups was compared by the student's $t$ test. For comparison of the seroprevalences of $T$. gondii infection between truck drivers and control subjects, the McNemar's test was used. We used the Pearson's chi-squared test and the two-tailed Fisher's exact test (when values were less than 5) to determine the association of $T$. gondii seropositivity and the characteristics of the drivers studied. Odds ratio (OR) and $95 \%$ confidence interval (CI) were calculated by multivariate analysis using logistic regression with the Enter method. Variables were included in the multivariate analysis if they had a $P$ value less than 0.05 in the bivariate analysis. The Hosmer-Lemeshow goodness of fit test was used to assess the fitness of our regression model. Statistical significance was set at a $P$ value less than 0.05 .

\section{Ethical aspects}

This study was approved by the Institutional Ethical Committee of the General Hospital of the Secretary of Health in Durango City. The purpose and procedures of the study were explained to all participants, and a written informed consent was obtained from each participant. Participants did not receive financial compensation.

\section{Results}

Anti-T. gondii IgG antibodies were found in 23 (12.0\%) of 192 interstate truck drivers and in 13 (6.8\%) of 192 controls. The difference between the seroprevalences in cases and controls was statistically significant according to the McNemar's pair test $(\mathrm{OR}=21.0$; 95\% CI: 1.23 358.38; $P=0.002)$. Of the 23 anti- $T$. gondii IgG positive drivers, 11 (5.7\%) had IgG levels higher than $150 \mathrm{IU} / \mathrm{ml}$, $1(0.5 \%)$ between 100 to $150 \mathrm{IU} / \mathrm{ml}$, and $11(5.7 \%)$ between 8 to $99 \mathrm{IU} / \mathrm{ml}$. In contrast, of the 13 anti-T. gondii IgG positive controls, 8 (4.2\%) had IgG levels higher than $150 \mathrm{IU} / \mathrm{ml}, 1$ (0.5\%) between 100 to $150 \mathrm{IU} / \mathrm{ml}$, and 4. (2.1\%) between 8 to $99 \mathrm{IU} / \mathrm{ml}$. Prevalence of high (>150 IU/ml) anti-T. gondii IgG levels was comparable among drivers and controls $(P=0.48)$. Anti- $T$. gondii IgM antibodies were found in $7(3.6 \%)$ cases and in 7 (3.6\%) controls $(P=1.00)$.

The socio-demographic characteristics among Toxoplasma-positive and Toxoplasma-negative drivers were not significantly different (Table 1). Of the work characteristics, only two variables showed potential association with seropositivity by bivariate analysis: "trips to the south of Mexico" $(P=0.007)$ and "number of eating
Table 1 Socio-demographic characteristics of truck drivers and seroprevalence of $T$. gondii infection

\begin{tabular}{|c|c|c|c|c|}
\hline \multirow[b]{3}{*}{ Characteristic } & \multirow{3}{*}{$\begin{array}{l}\text { Subjects tested } \\
\text { No. }\end{array}$} & \multirow{2}{*}{\multicolumn{2}{|c|}{$\frac{\text { Prevalence of }}{\text { T. gondii infection }}$}} & \multirow{3}{*}{$P$ value } \\
\hline & & & & \\
\hline & & No. & $\%$ & \\
\hline \multicolumn{5}{|l|}{ Age groups (years) } \\
\hline 30 or less & 21 & 1 & 4.8 & 0.25 \\
\hline $31-50$ & 120 & 13 & 10.8 & \\
\hline$>50$ & 51 & 9 & 17.6 & \\
\hline \multicolumn{5}{|l|}{ Sex } \\
\hline Male & 187 & 22 & 11.8 & 0.47 \\
\hline Female & 5 & 1 & 20.0 & \\
\hline \multicolumn{5}{|l|}{ Birth place } \\
\hline Durango State & 104 & 9 & 8.7 & 0.12 \\
\hline Other Mexican state & 88 & 14 & 15.9 & \\
\hline \multicolumn{5}{|l|}{ Residence } \\
\hline Durango State & 116 & 11 & 9.5 & 0.18 \\
\hline Other Mexican state & 76 & 12 & 15.8 & \\
\hline \multicolumn{5}{|l|}{ Residence area } \\
\hline Urban & 149 & 20 & 13.4 & 0.41 \\
\hline Suburban & 36 & 2 & 5.6 & \\
\hline Rural & 7 & 1 & 14.3 & \\
\hline \multicolumn{5}{|l|}{ Educational level } \\
\hline No education & 2 & 1 & 50.0 & 0.36 \\
\hline 1-6 years & 71 & 7 & 9.9 & \\
\hline 6-12 years & 113 & 14 & 12.4 & \\
\hline$>12$ years & 6 & 1 & 16.7 & \\
\hline \multicolumn{5}{|l|}{ Socio-economic level } \\
\hline Low & 38 & 7 & 18.4 & 0.37 \\
\hline Medium & 153 & 16 & 10.5 & \\
\hline High & 1 & 0 & 0.0 & \\
\hline
\end{tabular}

places during trips" $(P=0.01)$. Concerning clinical characteristics of drivers, the seroprevalence of $T$. gondii infection was higher in drivers with reflex impairment than in those without this impairment $(4 / 13,30.8 \%$ vs $19 / 179,10.6 \%$, respectively; $P=0.05$ ), and in drivers with hearing impairment than in those without this impairment $(3 / 7,42.9 \%$ vs $20 / 185,10.8 \%$, respectively; $P=$ 0.03). In the subset of 62 drivers with traffic accidents, the frequency of $T$. gondii infection was significantly higher in drivers with reflexes impairment than in those without this impairment $(3 / 5,60 \%$ vs $7 / 57,12.3 \%$, respectively; $P=0.02$ ). In the same subset of drivers with traffic accidents, the frequency of $T$. gondii infection was similar in drivers with hearing impairment than in those without this impairment $(0 / 1,0 \%$ vs $10 / 61,16.4 \%$, respectively; $P=1.0)$. Other clinical characteristics assessed were not associated with $T$. gondii seropositivity. 
Of the behavioral characteristics, only seven variables showed potential association with $T$. gondii seropositivity by bivariate analysis ( $P$ values $<0.05$ ): consumption of meat from goat, rabbit, horse, armadillo, iguana, snake and type of flooring at home. Multivariate analysis of work and behavioral characteristics of truck drives with $P$ value $<0.05$ obtained in bivariate analysis showed two positive associations of $T$. gondii exposure including trips to the south of Mexico (OR $=3.11$; 95\% CI: 1.02 9.44; $P=0.04)$ and consumption of horsemeat $(\mathrm{OR}=$ 5.18; 95\% CI: 1.62-16.55; $P=0.005$ ) (Table 2). The result of the Hosmer-Lemeshow test $(P=0.47)$ indicated an acceptable fit of our regression model.

\section{Discussion}

Toxoplasmosis is a food-borne [15] and a water-borne disease [16]. The seroprevalence of infection with $T$. gondii differs from state to state or region in the general population dependent on the presence or absence of risk factors for the infection. People living in low seroprevalence areas therefore have an increased risk for infection when visiting high seroprevalence states. The frequent traveling to different regions could therefore represent a risk factor for infection with $T$. gondii in interstate truck drivers. In the present study, the seroprevalence of $T$. gondii infection (12\%) was significantly higher in interstate truck drivers than in control subjects of the general population of Durango City. This two-fold increase points towards an association of T. gondii infection with an occupation of interstate truck driver. To the best of our knowledge, this is the first age- and gender matched case control study that demonstrates this association.

We attempted to identify contributing factors for infection in the truck drivers. The number of variables assessed in the present study was large. However, the number of variables was reduced by performing firstly bivariate analysis of all variables followed by multivariate

Table 2 Multivariate analysis of selected characteristics of truck drivers and their association with $T$. gondii infection

\begin{tabular}{llll}
\hline Characteristic & $\begin{array}{l}\text { Odds } \\
\text { ratio }\end{array}$ & $\begin{array}{l}\text { 95\% confidence } \\
\text { interval }\end{array}$ & P value \\
\hline Trips to Southern Mexico & 3.11 & $1.02-9.44$ & 0.04 \\
Number of eating places & 2.39 & $0.84-6.76$ & 0.10 \\
$\begin{array}{l}\text { Consumption of meat from: } \\
\text { Goat }\end{array}$ & 2.51 & $0.71-8.84$ & 0.14 \\
Rabbit & 1.21 & $0.39-3.76$ & 0.73 \\
Horse & 5.18 & $1.62-16.55$ & 0.005 \\
Armadillo & 2.60 & $0.46-14.49$ & 0.27 \\
Iguana & 1.59 & $0.50-5.10$ & 0.42 \\
Snake & 1.98 & $0.68-5.76$ & 0.20 \\
Soil floor at home & 1.83 & $0.67-4.99$ & 0.23 \\
\hline
\end{tabular}

analysis of a small number of selected variables with potential association $(P<0.05)$ with $T$. gondii seropositivity. None of the socio-demographic characteristics of the drivers was associated with $T$. gondii seropositivity. Multivariate analysis of work and behavioral characteristics of drivers showed that $T$. gondii infection was associated with work trips to the south of Mexico and with consumption of horsemeat. The seroprevalence of $T$. gondii infection in the general population is higher in southern Mexican states than in northern Mexican states. Seroprevalences higher than $50 \%$ have been found in southern Mexican states [17]. In contrast, a 6.1\% seroprevalence of $T$. gondii infection in the general population in the northern Mexican city of Durango was found [14]. Differences in climatic conditions may contribute to a higher seroprevalence of $T$. gondii infection in southern Mexican states. Seroprevalence of T. gondii infection is higher in humid than in dry climates [18-20]. Durango has a dry climate while southern Mexican states have a more humid climate. Regarding the association of $T$. gondii infection with consumption of horsemeat, this is the first time we found such association in a Mexican population. Our result strengthens the epidemiological role of horsemeat as a source of $T$. gondii infection. A $6.1 \%$ seroprevalence of $T$. gondii infection in horses in Durango State was reported recently [21]. We are not aware of further studies on the seroprevalence of T. gondii infection in equids in other Mexican states. Viable $T$. gondii has been recovered from tissues of equids experimentally infected with parasite oocysts [22]. In addition, several cases of clinical toxoplasmosis due to consumption of horsemeat have been described $[23,24]$. None of the drivers studied referred to eat raw meat. Nevertheless, there is no certainty that tissue cysts, if present in the horsemeat consumed, were all inactivated by cooking. Several factors including temperature and time of cooking, and thickness of meat should be consider for inactivation of tissue cysts. In a study using infectivity bioassays in cats and mice, inactivation of tissue cysts in sheep meat was reached by cooking meat at $60^{\circ} \mathrm{C}$ or $100^{\circ} \mathrm{C}$ for 10 minutes [25]. Further research to determine the heating parameters for inactivation of tissue cysts in horsemeat is needed.

Infection with $T$. gondii has been associated with traffic accidents [8] and reflex impairment [26]. In the present study, drivers with traffic accidents had a higher, but not statistically significant, seroprevalence of $T$. gondii infection than drivers without traffic accidents. The lack of association of $T$. gondii infection and traffic accidents may be due to a small sample size of our study population. However, since reflexes impairment showed a borderline $(P=0.05)$ association with $T$. gondii exposure in the present study we further assess whether seropositivity to $T$. gondii in the subset of drivers with traffic 
accidents differ between drivers with reflexes impairment and those without this impairment. Remarkably, seroprevalence of $T$. gondii infection in the subset of drivers with traffic accidents was significantly higher in drivers with reflex impairment than in those without this impairment. Results thus suggest that infection with $T$. gondii might lead to reflexes impairment that in turn may contribute for traffic accidents. Chronic infection with $T$. gondii has been associated with slowing of neural processing speed [27], reduced psychomotor performance and changes in behavior [28]. In the present study, we also found that $T$. gondii infection was associated with hearing impairment. This impairment might also contribute for traffic accidents. Infection with $T$. gondii has been associated with hearing loss in congenital toxoplasmosis. This hearing loss is likely caused by inflammation induced by tachyzoites of $T$. gondii [29]. Congenital hearing loss has not always been associated with primary infection during pregnancy [30], and very little is known about hearing loss caused by $T$. gondii in adults. In a previous study in an ethnic group (Tepehuanos) in Durango, Mexico, we found a significantly higher seroprevalence of IgM antibodies against $T$. gondii in subjects suffering from hearing impairment than those without this impairment [31]. It is unclear whether infection with $T$. gondii may affect the inner ear. However, dizziness -a symptom that may reflect disease in the inner ear- has been associated with $T$. gondii infection in the ethnic group of Huicholes [32] and in migrant agricultural workers in Durango, Mexico [33]. Further research to elucidate the role of $T$. gondii infection in inner ear disease is needed.

\section{Conclusions}

Results of this first case-control study suggest that interstate truck drivers in Mexico are at increased risk for $T$. gondii infection, and that $T$. gondii exposure may impact the health of truck drivers. Contributing factors for $T$. gondii exposure such as horsemeat should be taken into account for the design of optimal prevention measures against $T$. gondii infection.

\section{Competing interests}

The authors declare that they have no competing interests.

\section{Authors' contributions}

CAE conceived and designed the study protocol, participated in the coordination and management of the study, performed the laboratory tests, data analysis, and wrote the manuscript. SJPV, MSJ, and LOBS obtained blood samples, applied questionnaires and performed the data analysis. JHT, LFSA, and ERS performed the data analysis. OL performed the data analysis, and wrote the manuscript. All authors read and approved the final manuscript.

\section{Acknowledgements}

This study was supported by Juarez University of Durango State, Mexico.

\section{Author details}

'Faculty of Medicine and Nutrition, Juárez University of Durango State, Avenida Universidad S/N, 34000 Durango, Dgo, Mexico. Institute for Scientific Research "Dr. Roberto Rivera Damm", Juárez University of Durango State, Avenida Universidad S/N, 34000 Durango, Durango, Mexico. ${ }^{3}$ General Hospital, Secretary of Health, Avenida 5 de febrero 220, 34000 Durango, Mexico. ${ }^{4}$ Institute for Microbiology and Hygiene, Campus Benjamin Franklin, Charité Medical School, Hindenburgdamm 27, D-12203 Berlin, Germany. ${ }^{5}$ Current address: Medical and Scientific Affairs, Roche Molecular Systems, Pleasanton, CA 94588, USA.

Received: 28 September 2014 Accepted: 23 January 2015 Published online: 05 February 2015

\section{References}

1. Dubey JP. Toxoplasmosis of Animals and Humans. 2nd ed. Boca Raton, Florida: CRC Press; 2009.

2. Montoya JG, Liesenfeld O. Toxoplasmosis. Lancet. 2004;363:1965-76.

3. Walker M, Zunt JR. Parasitic central nervous system infections in immunocompromised hosts. Clin Infect Dis. 2005;40:1005-15.

4. Balasundaram MB, Andavar R, Palaniswamy M, Venkatapathy N. Outbreak of acquired ocular toxoplasmosis involving 248 patients. Arch Ophthal. 2010;128:28-32.

5. Karasawa T, Shikata T, Takizawa I, Morita K, Komukai M. Localized hepatic necrosis related to cytomegalovirus and Toxoplasma gondii. Acta Pathol Jpn. 1981;31:527-34.

6. Torrey EF, Bartko JJ, Yolken RH. Toxoplasma gondii and other risk factors for schizophrenia: an update. Schizophr Bull. 2012;38:642-7.

7. Alvarado-Esquivel C, Urbina-Álvarez JD, Estrada-Martínez S, Torres-Castorena A, Molotla-de-León G, Liesenfeld O, et al. Toxoplasma gondii infection and schizophrenia: a case control study in a low Toxoplasma seroprevalence Mexican population. Parasitol Int. 2011;60:151-5.

8. Flegr J, Havlícek J, Kodym P, Malý M, Smahel Z. Increased risk of traffic accidents in subjects with latent toxoplasmosis: a retrospective case-control study. BMC Infect Dis. 2002;2:11.

9. Alvarado-Esquivel C, Torres-Castorena A, Liesenfeld O, Estrada-Martínez S, Urbina-Álvarez JD. High seroprevalence of Toxoplasma gondii infection in a subset of Mexican patients with work accidents and low socioeconomic status. Parasit Vectors. 2012;5:13.

10. Arling TA, Yolken RH, Lapidus M, Langenberg P, Dickerson FB, Zimmerman SA, et al. Toxoplasma gondii antibody titers and history of suicide attempts in patients with recurrent mood disorders. J Nerv Ment Dis. 2009;197:905-8.

11. Alvarado-Esquivel C, Sánchez-Anguiano LF, Arnaud-Gil CA, López-Longoria JC, Molina-Espinoza LF, Estrada-Martínez S, et al. Toxoplasma gondii infection and suicide attempts: a case-control study in psychiatric outpatients. J Nerv Ment Dis. 2013;201:948-52.

12. Alvarado-Esquivel C, Liesenfeld O, Torres-Castorena A, Estrada-Martínez S, Urbina-Alvarez JD, Ramos-de la Rocha M, et al. Seroepidemiology of Toxoplasma gondii infection in patients with vision and hearing impairments, cancer, HIV, or undergoing hemodialysis in Durango, Mexico. J Parasitol. 2010;96:505-8.

13. Alvarado-Esquivel C, Torres-Berumen JL, Estrada-Martínez S, Liesenfeld O, Mercado-Suarez MF. Toxoplasma gondii infection and liver disease: a case-control study in a northern Mexican population. Parasit Vectors. 2011:4:75.

14. Alvarado-Esquivel C, Estrada-Martínez S, Pizarro-Villalobos H, Arce-Quiñones M, Liesenfeld O, Dubey JP. Seroepidemiology of Toxoplasma gondii infection in general population in a northern Mexican city. J Parasitol. 2011;97:40-3.

15. Tenter AM. Toxoplasma gondii in animals used for human consumption. Mem Inst Oswaldo Cruz. 2009;104:364-9.

16. Dubey JP. Toxoplasmosis - a waterborne zoonosis. Vet Parasitol. 2004;126:57-72.

17. Velasco-Castrejón O, Salvatierra-Izaba B, Valdespino JL, Sedano-Lara AM, Galindo-Virgen S, Magos C, et al. Seroepidemiology of toxoplasmosis in Mexico. Salud Publica Mex. 1992;34:222-9.

18. Julvez J, Magnaval JF, Meynard D, Perie C, Baixench MT. Seroepidemiology of toxoplasmosis in Niamey, Niger. Med Trop (Mars). 1996;56:48-50.

19. Assmar M, Amirkhani A, Piazak N, Hovanesian A, Kooloobandi A, Etessami R. Toxoplasmosis in Iran. Results of a seroepidemiological study. Bull Soc Pathol Exot. 1997;90:19-21. 
20. Markovich MP, Shohat T, Riklis I, Avni R, Yujelevski-Rozenblit D, Bassal R, et al. Seroepidemiology of Toxoplasma gondii infection in the Israeli population. Epidemiol Infect. 2014;142:149-55. doi:10.1017/S0950268813000903.

21. Alvarado-Esquivel C, Rodríguez-Peña S, Villena I, Dubey JP. Seroprevalence of Toxoplasma gondii infection in domestic horses in Durango State, Mexico. J Parasitol. 2012;98:944-5. doi:10.1645/GE-3174.1.

22. Dubey JP. Persistence of encysted Toxoplasma gondii in tissues of equids fed oocysts. Am J Vet Res. 1985;46:1753-4.

23. Pomares C, Ajzenberg D, Bornard L, Bernardin G, Hasseine L, Darde ML, et al. Toxoplasmosis and horse meat, France. Emerg Infect Dis. 2011;17:1327-8. doi:10.3201/eid1707.101642.

24. Sobanski V, Ajzenberg D, Delhaes L, Bautin N, Just N. Severe toxoplasmosis in immunocompetent hosts: be aware of atypical strains. Am J Respir Crit Care Med. 2013;187:1143-5. doi:10.1164/rccm.201209-1635LE.

25. El-Nawawi FA, Tawfik MA, Shaapan RM. Methods for inactivation of Toxoplasma gondii cysts in meat and tissues of experimentally infected sheep. Foodborne Pathog Dis. 2008:5:687-90. doi:10.1089/fpd.2007.0060.

26. Alvarado-Esquivel C, Estrada-Martínez S, Liesenfeld O. Toxoplasma gondi infection in workers occupationally exposed to unwashed raw fruits and vegetables: a case control seroprevalence study. Parasit Vectors. 2011;4:235 doi:10.1186/1756-3305-4-235.

27. Pearce BD, Hubbard S, Rivera HN, Wilkins PP, Fisch MC, Hopkins MH, et al. Toxoplasma gondii exposure affects neural processing speed as measured by acoustic startle latency in schizophrenia and controls. Schizophr Res. 2013;150:258-61. doi:10.1016/j.schres.2013.07.028.

28. Flegr J. Effects of Toxoplasma on human behavior. Schizophr Bull. 2007;33:757-60.

29. Salviz M, Montoya JG, Nadol JB, Santos F. Otopathology in congenital toxoplasmosis. Otol Neurotol. 2013;34:1165-9. doi:10.1097/ MAO.0b013e31828297b6.

30. Austeng ME, Eskild A, Jacobsen M, Jenum PA, Whitelaw A, Engdahl B. Maternal infection with Toxoplasma gondii in pregnancy and the risk of hearing loss in the offspring. Int J Audiol. 2010;49:65-8. doi:10.3109/ 14992020903214053

31. Alvarado-Esquivel C, Estrada-Martínez S, García-López CR, Rojas-Rivera A Sifuentes-Álvarez A, Liesenfeld O. Seroepidemiology of Toxoplasma gondii infection in Tepehuanos in Durango, Mexico. Vector Borne Zoonotic Dis. 2012;12:138-42. doi:10.1089/vbz.2011.0747.

32. Alvarado-Esquivel C, Pacheco-Vega SJ, Hernández-Tinoco J, Sánchez-Anguiano LF, Berumen-Segovia LO, Rodríguez-Acevedo FJ, et al. Seroprevalence of Toxoplasma gondii infection and associated risk factors in Huicholes in Mexico. Parasit Vectors. 2014;7:301. doi:10.1186/1756-3305-7-301.

33. Alvarado-Esquivel C, Campillo-Ruiz F, Liesenfeld O. Seroepidemiology of infection with Toxoplasma gondii in migrant agricultural workers living in poverty in Durango, Mexico. Parasit Vectors. 2013;6:113. doi:10.1186/1756-3305-6-113.

\section{Submit your next manuscript to BioMed Central and take full advantage of:}

- Convenient online submission

- Thorough peer review

- No space constraints or color figure charges

- Immediate publication on acceptance

- Inclusion in PubMed, CAS, Scopus and Google Scholar

- Research which is freely available for redistribution 